\title{
Effect of basal medium on growth and polyphenols accumulation by Gardenia jasminoides Ellis cell suspension
}

\author{
Gergana Krasteva ${ }^{1 *}$ \\ ${ }^{1}$ Laboratory of Cell Biosystems, The Stephan Angeloff Institute of Microbiology, Bulgarian Academy of Sciences, Plovdiv, Bulgaria
}

\begin{abstract}
The goal of this study was to analyze the effect of different medium bases on accumulation of biomass and secondary metabolites production by Gardenia jasminoides Ellis. cell suspension culture. The result revealed that different media have significant effect on biomass accumulation and production of polyphenol compounds. HPLC analyses showed that the higher polyphenol content was achieved when the cells were cultivated on McCOWN Woody plant Medium (WP) media for 14 days on darkness, at $24^{\circ} \mathrm{C}$. At this conditions, the cells produced maximal amounts of catechin $(24.44 \pm 2.46 \mu \mathrm{g} / \mathrm{g} \mathrm{DW})$, chlorogenic acid $(20.26 \pm 3.68 \mu \mathrm{g} / \mathrm{g} \mathrm{DW})$, epicatechin $(70.44 \pm 7.46 \mu \mathrm{g} / \mathrm{g} \mathrm{DW})$ and rutin $(45.90 \pm 0.26 \mu \mathrm{g} / \mathrm{g} \mathrm{DW})$. Both MS and WP media were optimal for biomass accumulation. When cultivated on these media, Gardenia cells accumulated maximal amount of dry biomass $\mathrm{ADB}=0.99 \pm 0.1 \mathrm{~g} / 100 \mathrm{ml}$ and $\mathrm{ADB}=0.93 \pm 0.04 \mathrm{~g} / 100 \mathrm{ml}$ for MS and WP, respectively. The corresponding growth indexes were GIDW $=1.68 \pm 0.2$ on MS and GIDW $=$ $1.58 \pm 0.07$ on WP medium. The reported results are the first step of future optimization of the nutrient medium composition and cultivation conditions essential for the scale - up of cultivation process of G. jasminoides cell suspension culture for mass production of polyphenolic compounds.
\end{abstract}

\section{Introduction}

Plants are extremely rich in various chemical compounds that play a crucial role in their growth and adaptation. These compounds are called secondary metabolites, some of which are phenols, terpenes, alkaloids and steroids. They have a great variety of biological activities and exceptional possibilities for application in the chemical-pharmaceutical industry, such as flavors, colorants, food additives $[1,2]$.

Plant biotechnology are promising method to obtaining secondary metabolites of commercial purposes $[3,4]$, offering a continuous renewable source through the cultivation of in vitro culture.

They have several pros:

1) the wanted product can be obtained everywhere by following the method of preparation and control]

2) does not depend on geographical or environmental conditions]

3) guarantees of pure plant material which is not contaminated with pesticides and herbicides; [5].

4) plant type at risk may be preserved for the future

In different phases of growth, the production of secondary metabolites is in different degrees. For example, in the exponential phase, the metabolites produced precursors for biomass production. There is published information that in the stationary phase most of the secondary metabolites are produced, which is important for different industries. An important point of working with in vitro cultures is to optimize the growing conditions so that for a short growth cycle to produce the optimal amount of biomass and secondary metabolites that are of crucial importance to the industry. This is related to a number of studies on the type of plant, the potential of the culture, different nutrient options, and the monitoring of biomass growth and metabolites accumulated in it.

Gardenia belongs to the genus Rubiaceae. It is grown in many areas in China. G. jasminoides is an evergreen shrub that grows from 60 to $155 \mathrm{~cm}$. with a number of applications in Chinese folk medicine, and very often as a cosmetic product or as a food coloring or in the textile industry.

In this work we are presenting the effect of basal medium on growth and polyphenols accumulation by Gardenia jasminoides Ellis cell suspension.

\section{Materials and methods}

\subsection{Plant material}

In this study, cell suspension culture of $G$. jasminoides obtained by leaves of nature plants with certified origins was used. The initiation of cell suspension culture is described [6]

Corresponding author: gergana.shk@gmail.com 


\subsection{Cultivation conditions}

Three types of basal media B5 (Camborg B5 Medium), WP (McCOWN Woody plant Medium) and MS (Murashiga and skoog Medium) were used. The media was supplemented with $30 \mathrm{~g} / \mathrm{L}$ sugar and 2NAA $0.5 \mathrm{BAP}$. For the needs of the experiments 14 days old culture was used as inoculum. The cell suspension was cultivated on orbital shaker $115 \mathrm{rpm}$ at $24^{\circ} \mathrm{C}$ in darkness. The subcultivated period was 14 days.

After the cultivation the biomass was collected and ADB and GIDW were calculated.

\subsection{Monitoring of growth}

The accumulated dry biomass was calculated on the equation below (1).

$$
\text { ADB }(\mathrm{g})=\text { Final dry - inoculum dry biomass }
$$

The growth index dry biomass was calculated on the equation (2)

$$
\mathrm{GIDW}=\frac{\mathrm{ADB}}{\text { Inoculum dry biomass }}
$$

Theэ dry content of the biomass was determined by using the direct method approximately $1 \mathrm{~g}$ (W1) of the samples in an oven at $50 \pm 2^{\circ} \mathrm{C}$ to obtain a constant weight (W2). The samples were removed from the oven, temperate in a desiccator and weighed. The results are expressed as a percentage of dry matter, as shown in the equation (3).

$$
\text { Weight }(\%)=\frac{\mathrm{W}_{1}-\mathrm{W}_{2}}{\mathrm{~W}_{1}} \times 100
$$

\subsection{Preparation of extracts}

Biomass from 14-day old Gardenia jasminoides cell suspension culture was dried and were powdered. Each sample was extracted in triplicate $(3 \times 10 \mathrm{ml})$ with $70 \%$ methanol, using approximately $0.3 \mathrm{~g}$ from dried biomass. The methanol was evaporated under vacuum on $50^{\circ} \mathrm{C}$ and after that we used solid phase extraction (SPE) - the columns was preconditioned according to manufacturer protocol (Sep-Pak ${ }^{\circledR}$ Clasic C18 Waters Corporation Milford, Massachusetts USA). The elution of polyphenols was done with $1 \mathrm{ml}$ HPLC grade methanol. This eluent was used for next HPLC experiments after filtration with syringe filters.

\subsection{HPLC analysis of phenolics}

Phenolics assays was performed by using Waters 1525 (Binary HPLC pump) and UV-VIS Waters 2487 (Dual $\lambda$ Absorbance Detector). A SUPELCO Analytical Discovery HS C18 column (25 cm x $4.6 \mathrm{~mm}, 5 \mu \mathrm{m})$
(SIGMA-ALDRICH) was used. The injected sample was $20 \mu \mathrm{L}$. Elution of compound was performed by using gradient of $1 \%$ acetic acid in water (Mobile phase A) and methanol (Mobile phase B) at speed $1 \mathrm{ml}$ per minute.

The elution program was:

$1 \mathrm{~min}-36 \min 90 \% \mathrm{~A}$ and $10 \% \mathrm{~B}, 36 \mathrm{~min}-37 \mathrm{~min}$ $78 \% \mathrm{~A}$ and $22 \% \mathrm{~B}, 37 \mathrm{~min}-47 \mathrm{~min}-70 \% \mathrm{~A}$ and $30 \% \mathrm{~B}$, $47 \mathrm{~min}-58 \mathrm{~min} 60 \% \mathrm{~A}$ and $40 \% \mathrm{~B}, 58 \mathrm{~min}-59 \min 54 \%$ $\mathrm{A}$ and $46 \% \mathrm{~B}, 59 \mathrm{~min}-71 \mathrm{~min} 40 \% \mathrm{~A}$ and $60 \% \mathrm{~B}, 71$ $\min -72 \min -20 \% \mathrm{~A}$ and $80 \% \mathrm{~B}, 72 \mathrm{~min}-75 \min -90$ $\% \mathrm{~A}$ and $10 \% \mathrm{~B}$.

The detection was done at $\lambda=280 \mathrm{~nm} /$ gallic acid, protocatechuic acid, $(+)$-catechin, vanillic acid, syringic acid, (-)-epicatechin, p-coumaric acid, salicylic acid, hesperidin/ and $\lambda=360 \mathrm{~nm} / \mathrm{chlorogenic}$ acid, caffeic acid, ferulic acid, rutin, rosmarinic acid, quercetin, kaempferol/

\subsection{Statistics}

All presented results are means \pm standard deviations (SD) $(n=3)$. For statistical analyses were used Microsoft Excel and SigmaPlot 10.0.

\section{Results}

\subsection{Cultivation of in vitro suspension cultures on different basic media}

Cultivation was performed in Erlenmeyer flasks (1L) and a working volume of $200 \mathrm{ml}$, on a rotary shaker at

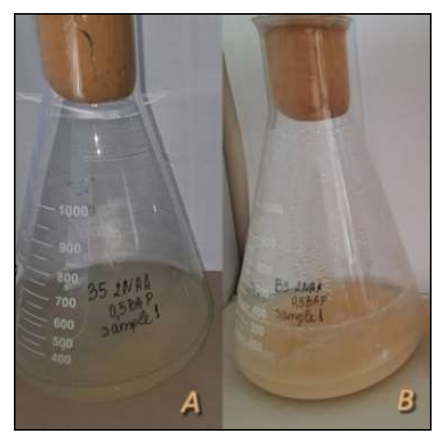

Fig. 1. Cell suspension culture from Gardenia jasminoides cultivated on media B5 with 2NAA $0.5 \mathrm{BAP}$ on day 0 (A) and day 14 (B)

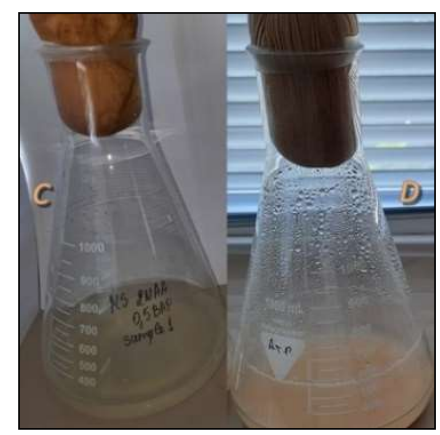

Fig. 2.

Suspension culture from Gardenia jasminoides cultivated on MS with 2NAA 0.5BAP media on day 0 (C) and day 14 (D) $115 \mathrm{rpm}$, at $24^{\circ} \mathrm{C}$ in the dark 14 days. For the experiments $30 \%$ inoculum 14-day old from cell suspension cultivated 
on 1/2MS 2NAA 0.5BAP supplemented with $30 \mathrm{~g} / \mathrm{L}$ sugar were used.

Picture of the cultures Fig. 1, Fig. 2 and Fig. 3.

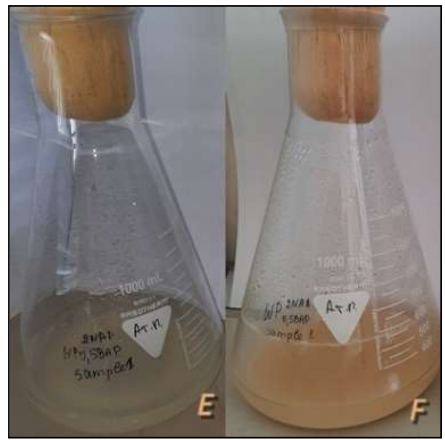

Fig. 3.

Cell

Suspension culture from Gardenia jasminoides cultivated on WP with 2NAA $0.5 \mathrm{BAP}$ on day 0 (E) and day 14 (F)

The results show that the highest value of dry biomass was achieved on MS $0.99 \pm 0.10 \mathrm{~g} / 100 \mathrm{ml}$, and WP with $0.93 \pm 0.03 \mathrm{~g} / 100 \mathrm{ml}$ (Fig. 4).

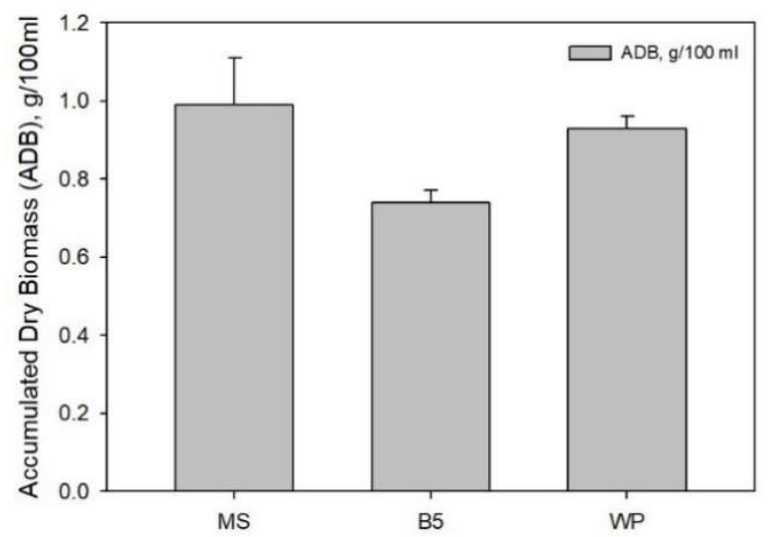

Fig. 4. Accumulated dry biomass (ADB), g/100ml, from cell suspension culture from Gardenia jasminoides cultivated on: $\mathrm{MS}$ - Murashiga and Skoog 2NAA + 0.5 BAP WP - McCOWN Woody plant Medium 2NAA $+0.5 \mathrm{BAP}$ B5 - Camborg B5 Medium 2NAA+0.5BAP

The highest growth index in the plant cell suspension cultivated on MS medium with growth regulators $2 \mathrm{NAA}$ and $0.5 \mathrm{BAP}-1.68 \pm 0.2 \mathrm{~g} / 100 \mathrm{ml}$, and the cell suspension culture cultivated under the same conditions, but on nutrient medium WP 2NAA and 0.5 BAP - $1.58 \pm 0.06$ g/100ml (Fig. 5)

3.2 Phytochemical profile to extracts from Gardenia jasminoides Ellis cell suspension culture, cultivated on different basal media - MS, B5, WP

HPLC analyzes clearly show that a higher polyphenol content is achieved when cells were cultured on Woody Plant McCOWN (WP) medium for 14 days, in darkness at $24^{\circ} \mathrm{C}$. Under these conditions, the cells produce maximum amounts of catechin $(24.44 \pm 2.46 \mu \mathrm{g} / \mathrm{g} \mathrm{DW})$, chlorogenic acid $(20.26 \pm 3.68 \mu \mathrm{g} / \mathrm{g} \mathrm{DW})$, epicatechin
$(70.44 \pm 7.06 \mu \mathrm{g} / \mathrm{g} \mathrm{DW})$, vanillic acid $(19.76 \pm 2.16 \mu \mathrm{g} / \mathrm{g})$ - Table 1.

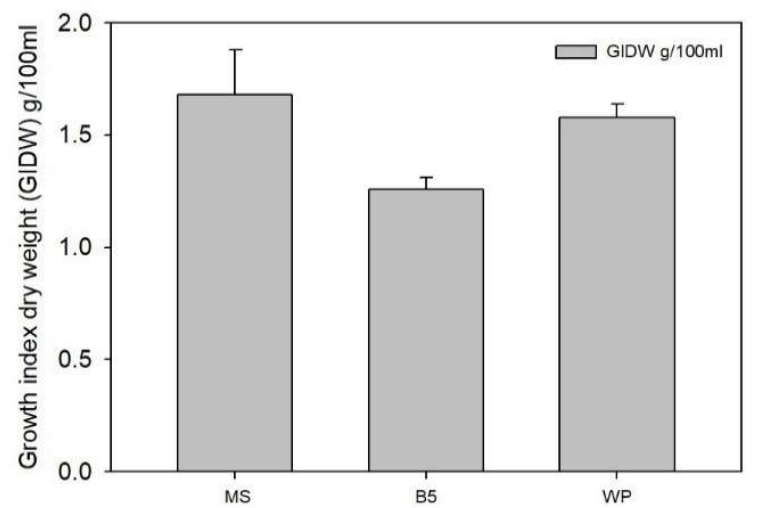

Fig. 5. Growth index dry weight (GIDW), g/100ml, from cell suspension culture from Gardenia jasminoides cultivated on: MS - Murashiga and Skoog 2NAA + 0.5 BAP

WP - McCOWN Woody plant Medium $2 \mathrm{NAA}+0.5 \mathrm{BAP}$ B5 - Camborg B5 Medium 2NAA+0.5BAP

The values of salicylic acid $11.43 \pm 1.9 \mu \mathrm{g} / \mathrm{g}$ DW, $12.72 \pm 0.79 \mu \mathrm{g} / \mathrm{g} \quad \mathrm{DW}$ and $13.93 \pm 1.19 \mu \mathrm{g} / \mathrm{g} \quad \mathrm{DW}$ respectively for MS, WP and B5 media (Table 1).

Table 1 HPLC quantification of polyphenolic content in extract from Gardenia jasminoides cell suspension culture in different media

\begin{tabular}{|c|c|c|c|}
\hline & MS day $14, \mu \mathrm{g} / \mathrm{g}$ & WP day 14, $\mu \mathrm{g} / \mathrm{g}$ & B5 day 14, $\mu \mathrm{g} / \mathrm{g}$ \\
\hline Gallic acid & ND & ND & ND \\
\hline Protocatehuic acid & ND & ND & ND \\
\hline (+)-Catechin & $36,54 \pm 19,47$ & $\mathbf{2 4 , 4 4} \pm 2,46$ & $34,08 \pm 0,78$ \\
\hline Chlorogenic acid & $9,89 \pm 0,91$ & $\mathbf{2 0 , 2 6} \pm 3,68$ & $16,34 \pm 3,49$ \\
\hline Vanillic acid & ND & $19,76 \pm 2,16$ & TRACE \\
\hline Caffeic acid & $13,43 \pm 0,01$ & $13,32 \pm 6,88$ & $27,78 \pm 9,14$ \\
\hline Syringic acid & $21,68 \pm 5,93$ & $18,82 \pm 4,56$ & $18,89 \pm 0,9$ \\
\hline (-)-Epicatechin & $51,56 \pm 13,31$ & $70,44 \pm 7,06$ & $68,32 \pm 13,67$ \\
\hline p-Coumaric acid & ND & TRACE & TRACE \\
\hline Ferulic acid & $21,45 \pm 0,65$ & $17,72 \pm 2.28$ & $13,16 \pm 2,01$ \\
\hline Salicylic acid & $11,43 \pm 1,9$ & $12,72 \pm 0,79$ & $13,93 \pm 1,19$ \\
\hline Rutin & TRACE & $45,90 \pm 0,26$ & ND \\
\hline Hesperidin & ND & ND & ND \\
\hline Rosmarinic acid & ND & $43,45 \pm 0,35$ & $46,94 \pm 0,12$ \\
\hline Quercetin & $54,28 \pm 0,6$ & $39,29 \pm 0,23$ & ND \\
\hline Kaempherol & ND & ND & ND \\
\hline
\end{tabular}

*ND - under the LOD

*TRACE - under the LOQ

\section{Discussion}

The aim of the study was to find the optimal basal media for the best growth and accumulation of polyphenols by Gardenia jasminoides cell suspension culture. The result for the ADB and GIDW were showed in Fig. 4 and Fig. 5. It was clear that when comparing the effect of 3 nutria medias, MS showed the highest amound of dry biomass and GIDW. It should be noted that B5 basal media showed the lowest values of ADB and GIDW. On the other hand, the difference between MS and WP was insignificant. The optimal conditions for the production of secondary metabolites and biomass growth are studies by number of scientists. Factors such as nitrate levels, type of nutriment medium are the basis for optimisation the growth [7] .

Next step of our study was the accumulation of polyphenols in biomass of Gardenia jasminoides cell suspension culture. It is known that secondary metabolites are produced as response of stress. On this base changes 
on nutritional components would have a significant effect on production second metabolites.

Our result in table 1 showed that when grow on nutrient WP cell suspension culture Gardenia jasminoides accumulated the highest amount of polyphenols. Interesting same of compounds like vanillic $\operatorname{acid}(19.76 \pm 2.16 \mu \mathrm{g} / \mathrm{g})$ and rutin $(45.90 \pm 0.26 \mu \mathrm{g} / \mathrm{g} \mathrm{DW})$ were found only in Gardenia jasminoides cell suspension culture cultivated on nutrient basal media WP. Vanillic acid is known to have a potential prevention to postmenopausal osteoporosis [4]. Rutin is very important metabolite for several pharmacological properties such as antioxidant activity, influence on diseases such as Alzheimer, diseases of the nervous system, for diabetes, diseases of the cardiovascular system and others [8].

On the other hand, some of the components were found in WP with the highest value compared to the same components to the other nutrient media. For example, chlorogenic acid $(20.26 \pm 3.68 \mu \mathrm{g} / \mathrm{g} \mathrm{DW})$ was with highest concentration on WP nutrient media, also epicatechin $(70.44 \pm 7.06 \mu \mathrm{g} / \mathrm{g} \mathrm{DW})$. It gives the impression that rutin and quercetin are not detected only in B5 basal media. The results clearly showed that gallic acid, protocatechuic acid, hesperidin and kaempferol were not detected anywhere.

Monford et al. [9] studied the change of shoot cultures of Ocimum basilicum on different culture media and strength MS on production of volatile fraction composition. It was found that different concentrations of even the same nutrient medium have various effects on growth. Analyzing the results, it was noticed that the concentration of vanillic acid is highest in the cell suspension of nutrient medium WP and accumulated only there.

One of the reasons why in vitro technologies are so preferred, is that absolutely preserve the biological species in nature! The use of biotechnological cultivation method in cosmetic industry is very interesting for many scientists today. This type of technology has good independent reproducibility of natural conditions as climate and altitude.

\section{Conclusions}

Plant cell cultures are source of a great number of phytochemicals. A large group of them are widely used in the manufacture of medicines and food supplements. Over the last decade, phytochemical elements obtained from in vitro cultures and their application in cosmetic products, were of great importance for the scientist [10].

The experiments performed in our study showed that the best base for cultivating Gardenia cell suspension culture was WP. The data obtained is the first step in the future optimization of the nutrient composition and culture conditions. It will be essential for scaling the process of cultivating $G$. jasminoides cell suspension culture for mass production of polyphenolic compounds.

In conclusion, the results are a good base for the future optimization of growth, the phytochemical properties and their future use as active cosmetic ingredients.

\section{References}

1. R. M. Cusidó, H. Vidal, A. Gallego, M. Abdoli, J. Palazón, Recent advances in pharmaceutical sciences III (Transworld Research Network, Kerala, 2013)

2. S. Gupta, D. Chauhan, ; K. Mehla, P. Sood, A. Nair, JBCP. 1, 55-62 (2010)

3. A. W. Alfermann, M. Petersen, Plant Cell Tiss. Organ. Cult. 43, 199-205 (1995)

4. T. Tanaka, H. Onuma, T. Shigihara, E. Kimura, Y. Fukuta, N. Shirasaka, T. Moriyama, Y. Homma, J. Biosci. Bioeng. 128, 622-629 (2019)

5. S. R. Rao, G. A Ravishankar, Biotechnol. Adv. 20, 101-153 (2002)

6. G. Krasteva, V. Georgiev, Proc. Eur. Fed. Biotech. (EFB, Barcelona, 2021)

7. H. N. Murthy, E.J. Lee, K.Y. Paek, Plant Cell Tiss. Organ. Cult. 118, 1-16 (2014)

8. A. Ganeshpurkar, K. Saluja Ajay, Saudi Pharmac. J. 25, 149-164 (2017)

9. L. Monfort, S. Kelly, S. Bertolucci, A. Lima, A. Alves de Carvalho, A. Mohammed, A. Blank, J. Pinto, Ind. Crops Prod. 116, 231-239 (2018)

10. G. Krasteva, V. Georgiev, A. Pavlov, Eng. Life Sci., 21, 68-76 (2021) 